This is the pre-peer reviewed version of the following article: "Taming Liquid Crystal Self-Assembly: The Multifaceted Response of Nematic and Smectic Shells to Polymerization" by JungHyun Noh, Benjamin Henx and Jan P.F. Lagerwall, Advanced Materials 2016, which has been published in final form at: http://onlinelibrary.wiley.com/doi/10.1002/adma.201603158/abstract. This article may be used for non-commercial purposes in accordance with Wiley Terms and Conditions for Self-Archiving.

\title{
Taming liquid crystal self-assembly: the multifaceted response of nematic and smectic shells to polymerization
}

\author{
JungHyun Noh, Benjamin Henx, and Jan P.F. Lagerwall* \\ Physics $\&$ Materials Science Research Unit, University of Luxembourg, \\ Luxembourg
}

E-mail: jan.lagerwall@lcsoftmatter.com

June 15, 2016

Liquid crystal-mediated self-assembly has emerged as a potent tool for advanced materials research and development, with particularly compelling opportunities opened by operating with topological defects. Demonstrated concepts range from nanoscopic assembly in defect lines [1-4] to actuators with specific folding or pumping actions. [5-8] Defects can be introduced in flat samples by advanced photopatterning, [9] but they in fact occur spontaneously in samples with spherical topology, like droplets or shells. If the director (the average molecule orientation) has a component in the spherical interface, the director field $\mathbf{n}(\mathbf{r})$ must contain topological defects, summing up to a total topological charge of +2 . [10] These curvature-induced defects are attractive e.g. in sensing: when threading nematic (orientational but no positional long-range order) droplets on biofibers, defect rings develop that reveal information on the fiber morphology, [11] and topological defects in nematic droplets enable detection of endotoxin with extraordinary sensitivity. [12] Another application avenue was proposed by Nelson: [13] because a tetrahedral defect arrangement minimizes the free energy of spherically symmetric nematic shells, they could generate colloidal particles with interactions directed for diamond-like colloid crystallization, of large interest for the photonics industry. However, when the first nematic shells were realized experimentally, [14] it turned out that the free energy is further reduced by breaking spherical symmetry, collecting all defects in a localized region with minimum shell thickness. Although the tetrahedral arrangement can be recovered by making the shell very thin, [15] this illustrates the challenge in harnessing liquid crystal-mediated self-assembly. The large parameter space is a blessing through the many possible outcomes, but a curse through the difficulty in accounting for all of them. There is thus a clear need to identify a method to tame the self-assembly in liquid crystal shells, without restricting the opportunities.

Another serious problem is the limited shell lifetime, on the order of days or less. Adjacent shells coalesce into a droplet and isolated shells collapse due to diffusion of the 
surrounding isotropic phases through the shell. [16] Moreover, while the temperature sensitivity of the defect arrangement in shells undergoing a nematic $(\mathrm{N})$ to smectic-A ( $\mathrm{SmA}$; 1D positional order) transition conveniently allows tuning of the defect configuration, [17-19] a fact we will be taking advantage of below, it also means that uncontrolled temperature variations can trigger undesired changes in the types and arrangements of defects. In any attempt to apply liquid crystal shells a means to ensure long-term shell stability and render a desired defect configuration permanent, is required.

In this paper we show that photopolymerization of reactive mesogens (RMs) [20, 21] in liquid crystal shells solves both problems, without removing the responsiveness that makes liquid crystals so useful in sensing and other applications. Even with low RM concentration, a certain defect configuration can be fixed and the shell lifetime dramatically extended. Interestingly, even if the polymerization is localized, the spherical topology ensures that $\mathbf{n}(\mathbf{r})$ is locked in place everywhere. Moreover, when carrying out the polymerization at a temperature near either boundary of the $\mathrm{N}$ phase range, we surprisingly find that the process induces a transition into the adjacent phase.

Using capillary microfluidics we prepare planar-aligned shells based on the commonly studied compound $8 \mathrm{CB}$. With $5 \%$ reactive mesogen RM257 and a low concentration of photoinitiator the phase sequence is $\mathrm{SmA} 29.1 \mathrm{~N} 42.0$ Iso. $/{ }^{\circ} \mathrm{C}$. At $35.0^{\circ} \mathrm{C}$, in the middle of the $\mathrm{N}$ phase, four closely spaced $+1 / 2$ defects are seen at the thinnest, bottom, part of the shell (Fig. 1b). We illuminate from above with UV light to initiate polymerization. Fig. $1 \mathrm{~b}$ shows that the texture on the bottom half of the shell is retained after polymerization, with identical defect arrangement. The top half of the shell now exhibits slight scattering from small grains. We will come back to their origin below.

We heat the polymerized shell over the clearing point $T_{N I}$ of $8 \mathrm{CB}$ and then cool it back to the N phase, see Fig. S3 and Movie 1 in the Supporting Information (SI). At $42.5^{\circ} \mathrm{C}$ the defect texture disappears and the shell appears mainly isotropic (Fig. S3b). However, by rotating the crossed polarizers by $45^{\circ}$ (Fig. S3c) we see that the birefringence, $\Delta n$, is still high, although $8 \mathrm{CB}$ is isotropic at this temperature. This shows that the polymer network has formed primarily on the top half of the shell, close to the UV light source. Here it has been templated into a uniform alignment by the $8 \mathrm{CB}$, which on the top half of the shell was in a defect-free nematic state at the temperature of polymerization.

Importantly, the fact that the defect pattern disappears on heating and that the lower half of the shell appears to have $\Delta n \approx 0$ demonstrates that the bottom half contains only a minor fraction of polymer network, allowing it to go fully isotropic upon heating. It is known from previous photopolymerization experiments [22-24] that the polymer network is concentrated towards the side closest to the UV light source. The gradient in UV intensity, as the photoinitiator absorbs light during passage through the sample, reduces the polymerization rate from top to bottom. The RMs are consumed more rapidly at the top, triggering diffusion from the bottom, and the polymer network thus develops more prominently at the top.

Although the defect pattern disappears on heating, as the bottom of the shell turns isotropic, the original nematic texture, with the defects positioned in the exact same 


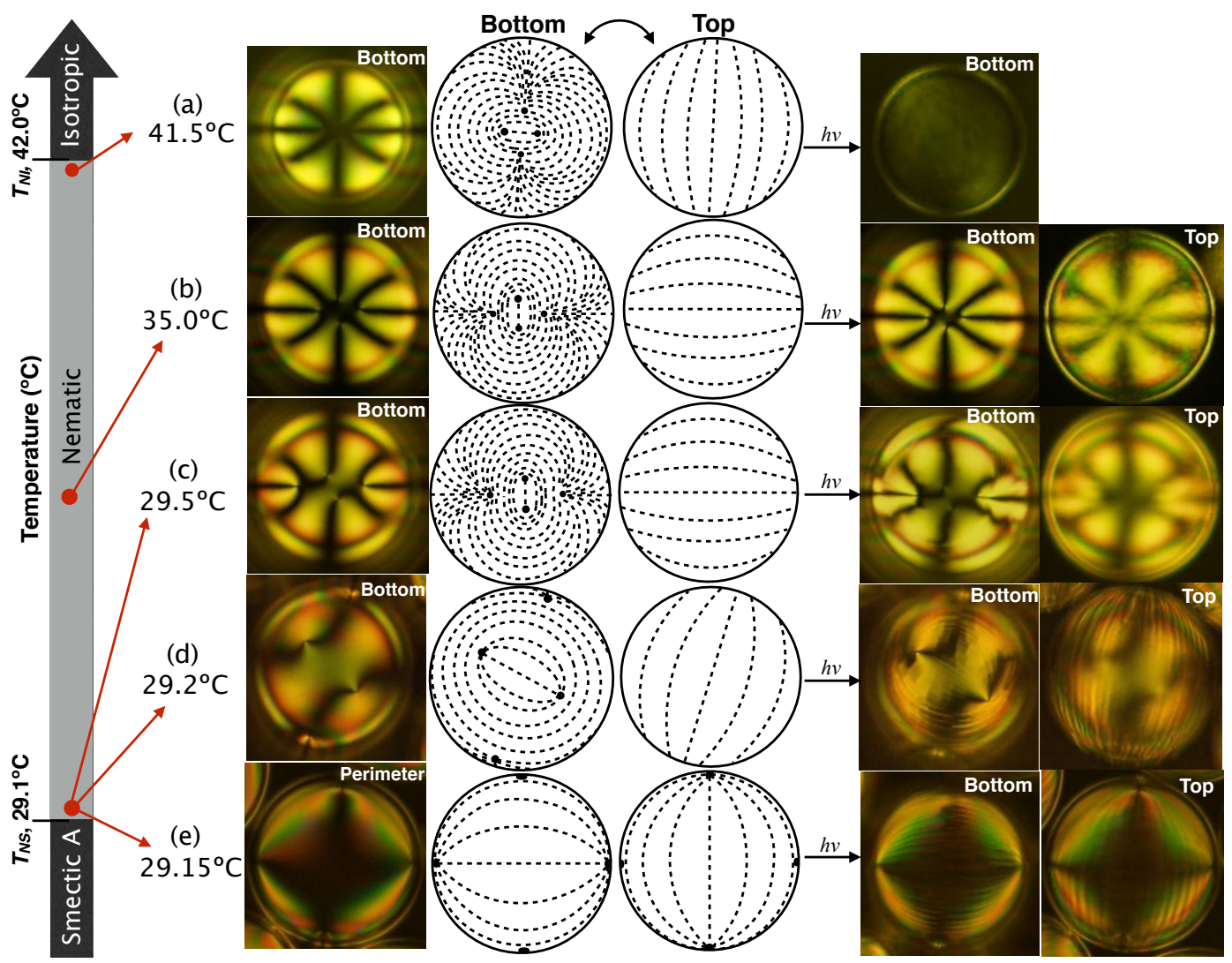

Figure 1: Polarizing microscopy textures of pristine 8CB+RM257 nematic shells (leftmost images) and schematic drawings of $\mathbf{n}(\mathbf{r})$ on the bottom and top surfaces, respectively, at different nematic temperatures. The right-most images show textures after polymerization at each temperature, with focus on the bottom and top of the shell, respectively. Shell diameters are $120-150 \mu \mathrm{m}$, the thickness below $5 \mu \mathrm{m}$. 
places as before, is recovered on cooling back to $35^{\circ} \mathrm{C}$ (Fig. S3d in SI). This confirms that the defects are successfully locked into place. This is remarkable since the bottom half of the shell, where the defects are located, contains so little polymer network that it turns isotropic on heating. In other words, thanks to the spherical shell topology it is enough to imprint a memory of $\mathbf{n}(\mathbf{r})$ on the defect-free side of the shell to control the defect arrangement on the other side.

Using another set of shells, we again polymerize in the nematic phase but at $41.5^{\circ} \mathrm{C}$, close to $T_{N I}$ (Fig. 1a). Before polymerization the texture is similar to that in Fig. 1b, but this time, immediately upon UV irradiation, the shell turns black between crossed polarizers, indicating a transition to an isotropic state (Fig. S4a-c in SI). Broer and coworkers reported isotropization upon photopolymerization of flat nematics near $T_{N I}$. [25] The heat released from the polymerization reaction elevates the temperature above $T_{N I}$. Without nematic order guiding the further chain growth, the polymer develops in a disordered fashion. Indeed, when cooling back to $35^{\circ} \mathrm{C}$ countless domains appear in our shell (Fig. S4d and Movie 2 in SI). The randomly formed polymer network now templates an unnatural mosaic-like configuration of the $\mathrm{N}$ phase.

If we instead cool the pristine nematic shells towards $T_{N S}$ prior to initiating polymerization, the four defects start moving up towards the equator. This is due to the divergence of the bend elastic constant $K_{3}$ upon approaching the transition to the SmA phase, and the fact that all defects can be pure splay defects only if they are arranged on a great circle. $[17,18,26]$ When polymerization is initiated by UV illumination, the defects remain roughly in place but they are somewhat blurred and the texture in the defect vicinity is distorted. As seen in Fig. 1c, the black brushes around the defects lose their smooth appearance and a light stripe formation is seen on the bottom side.

Carrying out the polymerization very close to $T_{N S}$, where the defects are even closer to the equator, a more drastic change in texture takes place, see Fig. 1d-e. Unexpectedly, upon UV initiation of the polymerization, a regular stripe pattern (spherical lunes) now develops all around the shell. Especially after polymerization at $29.15^{\circ} \mathrm{C}$ this pattern is highly regular. By refocusing between top and bottom we see that the lunes run parallel to the original $\mathbf{n}(\mathbf{r})$ (Fig. 1e).

The defect movement and the lune patterns observed during polymerization close to $T_{N S}$ remind us of the textural development in $8 \mathrm{CB}$ shells during cooling through the NSmA phase transition. $[17,18]$ The lune pattern signifies a buckling instability triggered by the incompatibility between smectic order and spherical shell geometry. The appearance of this texture in our experiments suggests that polymerization induces smectic order. We note that any heating effect from polymerization, driving the shell in Fig. 1a past $T_{N I}$, must still be active. Thus, the smectic phase is induced by polymerization although the temperature is in fact increasing.

We propose a scenario for the smectic-inducing effect, illustrated schematically in Fig. 2. The asymmetric design of $n$ CB-based mesogens leads to antiparallel association into dimers. [27] As illustrated in Fig. 2a, the length of such an 8CB dimer (3.56 nm) matches that of the RM257 mesogen $(3.6 \mathrm{~nm})$. We assume that the RMs are initially uniformly distributed within the $8 \mathrm{CB}$ host, orienting along $\mathbf{n}(\mathbf{r})$ (Fig. 2c). On cooling 
(a)

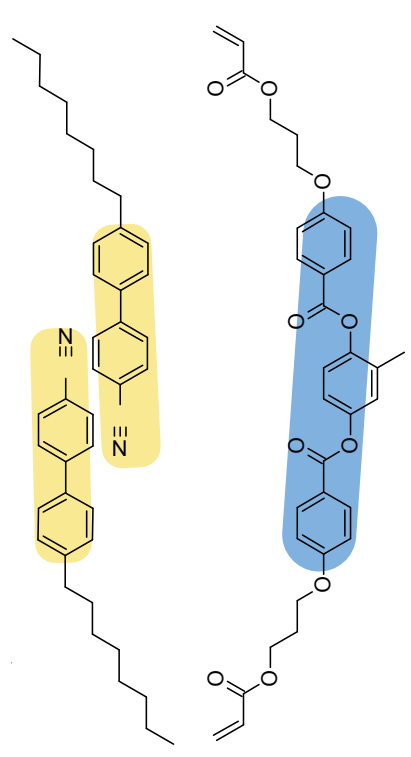

(c)

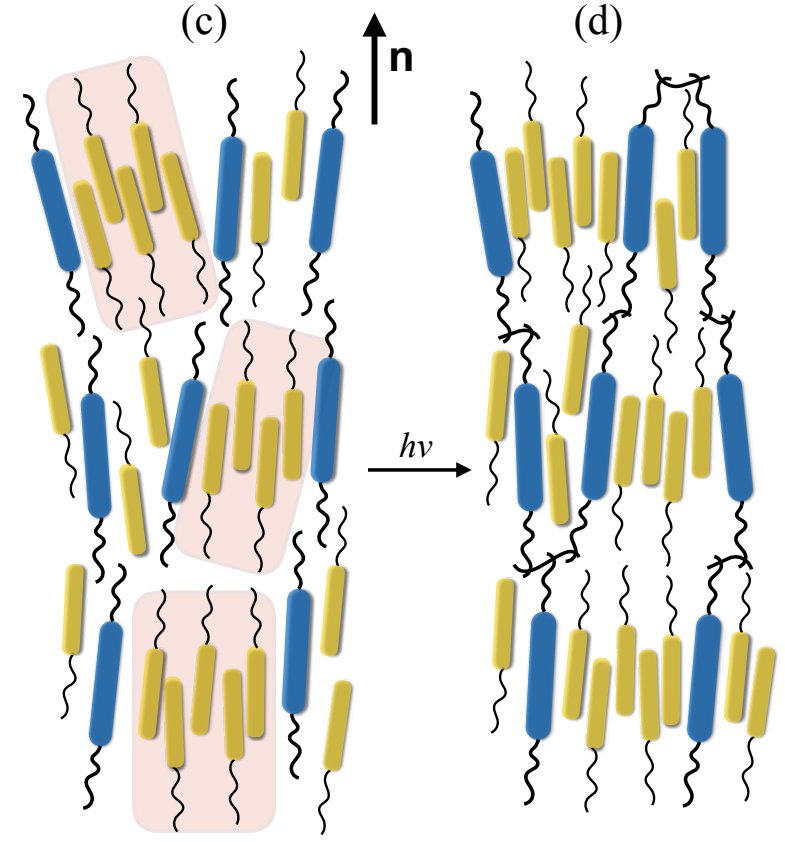

Figure 2: (a-b) Chemical structures of dimerized 8CB and RM257, with highlighted core regions. (c-d) Schematic drawing of a possible scenario during photopolymerization; (c) 8CB forms pre-smectic clusters (red) upon approaching the N-SmA transition. (d) Upon photopolymerization the cluster size diverges.

towards $T_{N S}, 8 \mathrm{CB}$ mesogens organize in a fluctuating smectic-like arrangement, forming cybotactic clusters. [28] When we initiate polymerization, chain propagation takes place perpendicular to $\mathbf{n}$, along a plane that effectively defines a smectic layer boundary. This is because the acrylate groups reacting to form the polymer are at each end of the RM257 monomer. Because of the geometric match between RM257 and the 8CB dimer, we believe that the propagation of the chains renders the cybotactic clusters permanent in time and extends their range in space to the point of divergence, thus breaking the translational symmetry on large scale and inducing the transition to SmA (Fig. 2d). In contrast, at high temperatures there are no cybotactic clusters, and the small fraction of RMs is not enough to propagate smectic order over long distances. Instead, polymerization then induces tiny smectic-like islands, probably the scattering grains in Fig. 1b.

We have repeatedly investigated a set of polymerized shells as a function of time, finding no change in shape or texture over the time scale of several months. We thus expect that the polymerization renders the shell truly long-term stable. Moreover, we test for temperature stability on cooling polymerized shells far below the normal liquid crystal temperature range (Fig. S8 in SI). The shells maintain their spherical shape without collapsing, as well as the locked-in nematic texture, confirming their excellent 
stability against strong temperature changes.

Finally, we also cool an 8CB+RM257 shell slowly into SmA before we initiate polymerization, such that the striped lune pattern characteristic of SmA shells can develop as an equilibrium texture, $[17,18]$ without any influence of polymer chain growth. When this pattern is stable, polymerization is initiated by UV illumination. This time, no further change is detected in the texture, see Fig. 3a-c and Movie 3 in the SI. As the shell is heated up through the original nematic and then isotropic phase ranges, the striped lune texture remains. The only traces of phase transitions are reductions in birefringence and the disappearance of some of the finest chevron modulation.

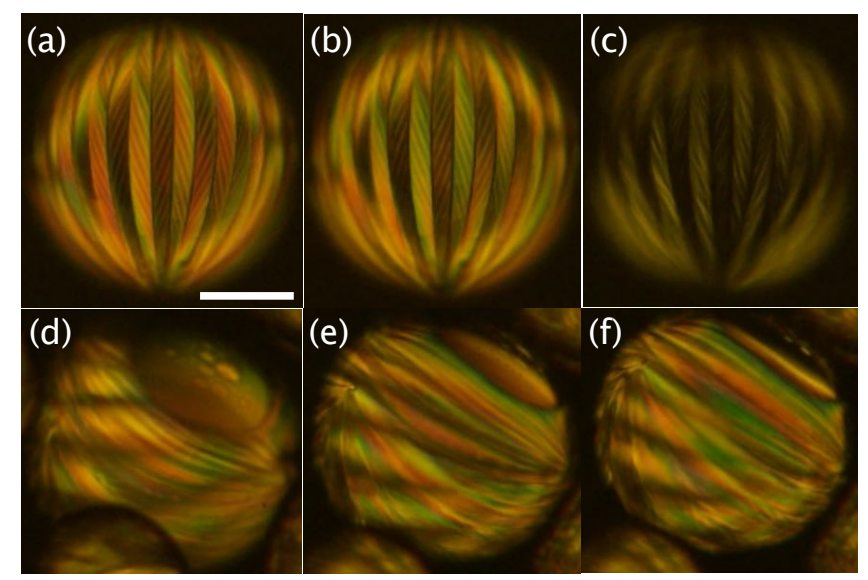

Figure 3: 8CB+RM257 shells polymerized in $\mathrm{SmA}$; (a) immediately after polymerization, (b) after heating to the $8 \mathrm{CB}$ nematic phase range, and (c) after heating to the isotropic range of $8 \mathrm{CB}$. (d) A polymerized SmA shell imaged just after flipping the capillary over. (e-f) Gravity rotates the shell back to its original orientation. Scale bar is $50 \mu \mathrm{m}$.

To image also the shell back side we rapidly flip a capillary, filled with a suspension of shells polymerized in the SmA phase, upside down, see Fig. 3d-f and Movie 4 in the SI. This provides striking further evidence of the fact that the polymerization takes place from the top. Because of the shell asymmetry the center of mass is now above the geometric center, and we can follow how gravity slowly rotates the shell back to its original orientation. The original shell bottom appears like an extremely thin patch that closes up the heavily striped polymerized part of the shell. In fact, the patch is so thin that it appears isotropic between crossed polarizers, the only birefringent areas being small islands which most likely are local areas of polymerized RM257.

We end the paper by discussing possible application opportunities. It is particularly attractive that we can localize polymerization to one half of the shell, allowing the other half to go through a major reorganization like a nematic-isotropic transition. Yet, as long as planar alignment prevails, the topologically required defects are locked into the configuration that was present at the temperature of polymerization. This is attractive in the context of bio-sensing, utilizing the same principles that so far were demonstrated 
with nematic droplets, $[11,12]$ where topological defects can dramatically enhance sensitivity. [12] The potential advantages of shells are manyfold. They can be much larger, yet with excellent control of the ground state alignment, thus simplifying texture analysis. Complex director fields can be induced by combining different alignment agents, allowing e.g. bend from inside to outside, inducing specific defect configurations. [29,30]

On the other hand, with optimized mixture components we can incorporate much larger fractions of RM, allowing us to take advantage of the structure formation driven by liquid crystalline self-assembly in the shell to create polymer materials with enhanced network morphology. For instance, the SmA shell polymerization locks the spherical lunes, and to some extent even the secondary modulation, into place, as seen by these patterns remaining upon heating beyond the clearing point of $8 \mathrm{CB}$. This means that we can easily obtain highly anisotropic polymer chain alignment, with a complex yet regular arrangement over macroscopic areas, from uniform alignment on the shell inside to a zigzag modulation on the shell outside. $[17,18]$ We would then have a curved polymer shell that to some extent resembles the eye's cornea, gaining exceptional mechanical strength from a radially periodic orientational modulation of its highly anisotropic collagen network. [31] It would be a very interesting future extension to measure the mechanical properties of the curved polymer films produced by polymerizing SmA shells containing a higher content of reactive mesogens. With respect to Nelson's proposed new colloidal chemistry, polymerization of the shells represents a major step forward. Our work shows that nematic shells can be made durable by polymerization, with the defects and their arrangement intact.

In conclusion, with photopolymerization of liquid crystal shells we can turn shortlived fluid objects of academic interest into long-term stable systems that are robust enough to be realistically considered for applications. If a planar-aligned nematic shell is polymerized at a temperature far away from phase transitions, the director field throughout the shell is preserved, with topological defects locked into the configuration adapted prior to polymerization. Surprisingly, this holds although the polymerization is concentrated mainly to the shell half that does not contain defects, due to localized initiation of polymerization. This is a new illustration of the power of spherical topology applied to liquid crystal self-assembly, opening for interesting applications in e.g. sensing. Since numerous options exist to further modify the structure within the shell prior to polymerization, e.g. by using chiral liquid crystals or by combining different aligning agents, polymerization of liquid crystal shells opens a vast and prolific playground for advanced materials design.

\section{Experimental section}

Full details are provided in the SI. Shells were produced using a coaxial glass capillary microfluidic set-up, [32] a mixture of water and glycerol (50/50 volume ratio) with 1 wt.- $\%$ of polyvinyl alcohol (PVA, Mw 13,000 - 23,000 $\mathrm{g} \mathrm{mol}^{-1}, 87-88 \%$ hydrolyzed) used for inner and outer phases. Density mismatch lowers the inner drop to the bottom of the shell, which is thus the thinnest point. Shell suspensions are filled into flat rectangular 
glass capillaries for polarizing optical microscopy (Olympus BX-51), the temperature controlled by a hot stage (Linkam T95-PE).

To avoid premature polymerization, a yellow-green filter was inserted in the microscope. Photopolymerization was initiated using a UVATA LED UV curing system (8800 $\mathrm{mW} / \mathrm{cm}^{2}$ at full power, $365 \mathrm{~nm}$ ). Illumination was at $45^{\circ}$ angle to the sample plane and about $3 \mathrm{~cm}$ above the sample. For fast polymerization the sample was exposed at maximum power for 1 minute, and for slow polymerization the power was reduced to $10 \%$ and the illumination time was 2 minutes.

\section{Acknowledgement}

We thank Merck Korea for the sample of RM257. Financial support from the Fonds National de la Recherche Luxembourg, PhD Grant 6992111, and the European Research Council (ERC, consolidator project INTERACT, grant code 648763), is gratefully acknowledged.

\section{References}

[1] X. Wang, Y. Kim, E. Bukusoglu, B. Zhang, D. Miller, N. Abbott. Phys. Rev. Lett. 2016, 116, 14147801.

[2] X. Wang, D. Miller, E. Bukusoglu, J. de Pablo, N. Abbott. Nat. Mater. 2015.

[3] E. Lee, Y. Xia, J. Ferrier, Robert C., H.-N. Kim, M. A. Gharbi, K. J. Stebe, R. D. Kamien, R. J. Composto, S. Yang. Adv. Mater. 2016, 28, 142731.

[4] H. Agha, Y. Galerne. Phys. Rev. E 2016, 93, 4.

[5] M. McConney, A. Martinez, V. Tondiglia, K. Lee, D. Langley, I. Smalyukh, T. White. Adv. Mater. 2013, 25, 415880.

[6] R. Kohlmeyer, Ryan, J. Chen. Angew. Chem. (Int. Ed.) 2013, 52, 359234.

[7] D. Liu, W. M. Bastiaansen, Cees, M. J. den Toonder, Jaap, J. Broer, Dirk. Angew. Chem. (Int. Ed.) 2012, 51, 4892.

[8] E.-K. Fleischmann, H.-L. Liang, N. Kapernaum, F. Giesselmann, J. P. F. Lagerwall, R. Zentel. Nat. Commun. 2012, 3 ARTN: 1178.

[9] Y. Guo, M. Jiang, C. Peng, K. Sun, O. Yaroshchuk, O. Lavrentovich, Q.-H. Wei. Adv. Mater. 2016, 28, 122353.

[10] T. Lopez-Leon, A. Fernandez-Nieves. Colloid Polym. Sci. 2011, 289, 4345.

[11] L. Aguirre, A. de Oliveira, D. Sec, S. Copar, P. Almeida, M. Ravnik, M. Godinho, S. Zumer. Proc. Natl. Acad. Sci. U. S. A. 2016, 113, 51174. 
[12] I.-H. Lin, S. Miller, Daniel, J. Bertics, Paul, J. Murphy, Christopher, J. de Pablo, Juan, L. Abbott, Nicholas. Science 2011, 332, 60351297.

[13] D. R. Nelson. Nano. Lett. 2002, 2, 101125.

[14] A. Fernandez-Nieves, V. Vitelli, A. Utada, D. R. Link, M. Marquez, D. R. Nelson, D. A. Weitz. Phys. Rev. Lett. 2007, 99, 15157801.

[15] T. Lopez-Leon, V. Koning, K. B. S. Devaiah, V. Vitelli, A. Fernandez-Nieves. Nat. Phys. 2011, 7391.

[16] J. Noh, K. Reguengo De Sousa, J. P. F. Lagerwall. Soft Matter 2016, 12, 2367 .

[17] H.-L. Liang, S. Schymura, P. Rudquist, J. Lagerwall. Phys. Rev. Lett. 2011, 106, 24247801.

[18] T. Lopez-Leon, A. Fernandez-Nieves, M. Nobili, C. Blanc. Phys. Rev. Lett. 2011, 106, 24247802 .

[19] D. Sec, T. Lopez-Leon, M. Nobili, C. Blanc, A. Fernandez-Nieves, M. Ravnik, S. Zumer. Phys. Rev. E 2012, 86 020705(R).

[20] I. Dierking, P. Archer. RSC Adv. 2013, 3, 4826433.

[21] D. J. Broer, J. Boven, G. N. Mol, G. Challa. Die Makromolekulare Chemie 1989, 190, 92255 .

[22] D. J. Broer, J. Lub, N. Mol. Nature 1995, 378467.

[23] C. L. van Oosten, D. Corbett, D. Davies, M. Warner, C. W. M. Bastiaansen, D. J. Broer. Macromolecules 2008, 41, 228592.

[24] S. Relaix, C. Bourgerette, M. Mitov. Liq. Cryst. 2007, 34, 91009.

[25] D. J. Broer, N. Grietje, N. Mol. Makromol. Chem. 1991, 19259.

[26] H. Shin, M. Bowick, X. Xing. Phys. Rev. Lett. 2008, 101, 3037802.

[27] D. Demus, J. W. Goodby, G. Gray, H.-W. Spiess, V. Vill, editors. Handbook of liquid crystals. Wiley-VCH, Weinheim, 1998.

[28] A. De Vries. Mol. Cryst. Liq. Cryst. 1970, 10219.

[29] H. Liang, J. Noh, R. Zentel, P. Rudquist, J. Lagerwall. Philos. Transact. A Math. Phys. Eng. Sci. 2013, 371, 198820120258.

[30] H.-L. Liang, R. Zentel, P. Rudquist, J. Lagerwall. Soft Matter 2012, 8, 205443.

[31] H. Aghamohammadzadeh, R. Newton, K. Meek. Structure 2004, 12, 2249.

[32] A. Utada, L. Chu, A. Fernandez-Nieves, D. Link, C. Holtze, D. Weitz. Mrs Bull 2007, 32, 9702 . 